\title{
BILATERAL FACIAL PALSY IN GUILLAIN-BARRE SYNDROME- INCIDENCE AND PROGNOSTIC SIGNIFICANCE
}

\author{
Ram Dayal Singh ${ }^{1}$, Kumar Abhinav²
}

${ }_{1}^{1}$ Associate Professor, Department of Internal Medicine, Patna Medical College and Hospital (PMCH), Patna.

2Junior Resident, Department of Internal Medicine, Patna Medical College and Hospital (PMCH), Patna.

ABSTRACT
BACKGROUND
Bilateral facial palsy is rare in clinical medicine, but it is common in Guillain-Barre syndrome (GBS). Hence, this prospective study
was conducted to see the incidence and prognostic significance of bilateral facial palsy in Guillain-Barre syndrome.

\section{MATERIALS AND METHODS}

21 established cases of GBS from different wards, central emergency and ICU of Patna Medical College, Patna, a tertiary care centre were selected for this study from Jan 2017 to June 2017.

\section{RESULTS}

The incidence of bilateral facial palsy in GBS was observed to be $14.2 \%$ in this study and all cases with bilateral facial palsy needed ventilator support indicating a poor prognosis. This was the most important observation. The mortality in this study was $14 \%$.

\section{CONCLUSION}

Though the sample size was small, but all cases with bilateral facial palsy needed ventilator support. Hence, all such cases should be monitored closely for the features of respiratory involvement during treatment.

\section{KEYWORDS}

Guillain-Barre Syndrome, Bilateral Facial Palsy, Facial Nerve.

HOW TO CITE THIS ARTICLE: Singh RD, Abhinav K. Bilateral facial palsy in Guillain-Barre syndrome- incidence and prognostic significance. J. Evolution Med. Dent. Sci. 2017;6(95):7051-7054, DOI: 10.14260/jemds/2017/1529

\section{BACKGROUND}

Guillain-Barre syndrome, a known cause of bilateral facial palsy, is an acquired immunologically mediated or autoimmune acute inflammatory demyelinating polyradiculopathy. ${ }^{1,2}$ It reaches peak disability within 4 wks. It is usually symmetrical and predominantly motor neuropathy which frequently involves facial nerves, often bilateral along with other cranial nerves. It is one of the most common cause of acute neuromuscular paralysis.1,2

In 1834, Wardrop and Ollivier described this type of paralysis, which is probably the earliest description.

In 1859, Landry reported acute ascending predominantly motor paralysis with respiratory failure leading to death. ${ }^{1}$

In 1960, Guillain-Barre and Strohl reported acute areflexic predominantly motor paralysis with albuminocytological dissociation in CSF in two shoulders. ${ }^{2}$ Electrophysiological changes were also defined.

In 1949, Haymaker and Kernohan published a paper entitled Landry Guillain-Barre syndrome, in which they upheld that the condition reported by Landry and GuillainBarre is the same. ${ }^{3}$

In 1956, Charles Miller Fisher reported a variant of GBS having external ophthalmoplegia, sluggish pupillary reflexes, ataxia and areflexia in three cases in which case was having

'Financial or Other Competing Interest': None.

Submission 26-07-2017, Peer Review 06-12-2017,

Acceptance 12-12-2017, Published 23-12-2017.

Corresponding Author:

Dr. Ram Dayal Singh,

Associate Professor,

Department of Internal Medicine,

Patna Medical College and Hospital (PMCH) Patna.

E-mail: dr_rdsingh@yahoo.in

DOI: $10.14260 /$ jemds $/ 2017 / 1529$ facial palsy. ${ }^{4}$ All three cases recovered spontaneously.

Finally, in 1978, National Institute of Neurological Disorder and Strokes (NINDS) defined the diagnostic criteria of GBS, which was later on modified by Asbury et al in 1999.5

The clinical manifestation in GBS are due to cell-mediated immunological reaction directed at peripheral nerves. Brostoff et al suggested a basic protein P2 in the myelin sheath of peripheral nerve acting as antigen. Antimyelin antibodies play an important role in initiating the disease process. A number of autoantibodies are found in the patients of GBS, most prominently being anti-GQ1b.

$15 \%$ cases have anti-GM1 antibody earlier in the course of disease. Many subtypes of GBS have been described- Acute Inflammatory Polyneuropathy (AIDP), Acute Motor Axonal Neuropathy (AMAN), Acute Motor Sensory Axonal Neuropathy (AMSAN), Miller-Fisher Syndrome (MFS), recurrent GBS, Pure sensory syndrome etc.

\section{There are well-defined Criteria for the Diagnosis of GBS}

A. Features mandatory for diagnosis-

1. Progressive motor weakness for more than one limb.

2. Areflexia.

B. Features strongly supporting the diagnosis: -

1. Progression up to 4 weeks.

2. Relative symmetry.

3. Mild sensory symptoms, but no sensory signs.

4. Cranial nerve involvement.

5. Autonomic dysfunction.

6. Absence of fever at the onset of neurologic symptoms.

7. Albuminocytological dissociation (cells less than 10/ mm3) in CSF.

8. Electrodiagnostic features. 
On the other hand, there are Features that Cast Doubts on the Diagnosis of GBS

1. Persistent asymmetry of weakness.

2. Persistent bowel and/or bladder dysfunction.

3. Bladder and bowel dysfunction at the onset.

4. More than 50 mononuclear cells/ mm3 in CSF.

5. Presence of Polymorph in CSF.

6. Sharp sensory level.

GBS is clinically prominent by acute onset and predominantly motor and areflexic paralysis of limbs. It runs a course of 1 to 4 weeks, during which neurological disabilities are completed.

The stage of maximal neurological disability is followed by a plateau phase lasting for one to several weeks, after which recovery begins. $1 / 3$ rd cases start improving within 15 days and next third after 2 weeks. Whereas most cases of last third show signs of recovery within 2 months. $70 \%-80 \%$ patients recover with little or no residual disability, but 7\% cases may remain severely handicapped. Mortality ranges from $4 \%-13 \%$.

\section{Predictors of Poor Outcomes are-}

1. Age over 40 years.

2. Rapid onset.

3. Requirement of ventilator support (Bilateral facial palsy is the predictor of need for ventilator support).

4. Electrophysiological criteria.

\section{Diagnosis of GBS is based on-}

1. Clinical picture.

2. CSF study.

3. Electrophysiological study.

\section{Electrophysiological Feature Includes}

a. Reduction of motor nerve conduction velocity in 2 or more nerves.

b. Prolonged distal motor latencies in 2 or more nerves.

c. Conduction block or abnormal temporal dispersion in 1 or more nerve. There should be more than $20 \%$ drop in peak amplitude of CMAP recorded proximally.

d. Absent of prolonged F-wave latencies in 2 or more motor nerves.

In addition to General Management, the Specific Treatment of GBS has Three Options

a. Steroid.

b. Plasma Exchange (PE).

c. IVIg (Intravenous Immunoglobulin).

The Steroid is Ineffective. The Plasma Exchange is an Important Modality of Treatment and is Indicated in Certain Conditions

- Cases reporting after several weeks of onset.

- $\quad$ Cases unable to walk unassisted (Grade 3).

- Cases with bulbar palsy.

- Cases who are at the verge of respiratory failure.

- Cases having rapid progression of the disease.

Plasma Exchange has many Benefits

- Rapid onset of action.

- $\quad$ Safe and well tolerated.
- $\quad$ Effective in 2/3rd of cases.

- Shorten time on ventilation.

- Decreases the percentage of residual disability.

\section{Disadvantages with Plasma Exchange are also Worth Mentioning}

- Not available at all centres.

- Needs special equipment and trained persons.

- Technically difficult in children.

- $\quad$ Repeated venous access.

IVIg therapy is simple and more popular and it was adopted in this study. A large International multicentric study ${ }^{6}$ has established that it is as effective as Plasma exchange. It contains polyvalent immunoglobulin $\mathrm{G}$ derived from large number of blood donors. Its mechanism of action is variable and complex. Possible actions are anti-idiotypic antibody effect, blockade of Fc receptor, down regulation of immunoglobulin production, down regulation of $\mathrm{B}$ cells, inhibition or attenuation of cytokine actions by anticytokine antibody, interference with T-cell activation, induction of suppressor T-cell and virus neutralisation.

Indication of IVIg are same as Plasma exchange, but it is preferred in children and elderly. It should be avoided in pregnancy and 2 weeks after the onset of GBS. It is effective in Miller-Fisher syndrome and acute pandysautonomia also. Dose of IVIG in adult is $400 \mathrm{mg} / \mathrm{kg} /$ day for 5 consecutive days. Its beneficial effects last for 2 - 9 weeks. It is usually safe and well tolerated, but some side effects associated with IVIG are low-grade fever with chills or flu-like syndrome, backache, tachycardia, aseptic meningitis, rash or eczema, alopecia, reversible neutropenia, haemolysis, immune complex arthritis, thromboembolism, DVT, transmission of Hep C virus, acute hypotension, CCF, proteinuria and renal failure.

There are certain Advantages with the use of IVIG and they are-

- Wide availability.

- No equipment needed.

- Can be given at any centre.

- Can be given in children and elderly.

- $\quad$ No risk of HIV transmission.

- $\quad$ Rapid response and 75\% cases show good response.

\section{Certain Disadvantages are-}

- All cases do not respond to IVIg.

- Allergic and anaphylactic reaction in patients with IgA deficiency.

- Important and dreaded complication of Guillain-Barre syndrome is respiratory failure, which develops insidiously and persists treacherously silent.

- Usually, 3 factors contribute to development of respiratory failure.

1. Lack of airway protection.

2. Respiratory muscle weakness.

3. Pulmonary complications.

Most important is that the case of Guillain-Barre syndrome should be monitored closely for the respiratory involvement. Facial nerve involvement along with bulbar dysfunction, 
autonomic involvement, reduced single breath count, reduced chest expansion and axonopathy are the parameters to be monitored for the development of respiratory involvement. Facial weakness was suspected on clinical ground and confirmed by NC Study. The autonomic involvement is most likely as a result of direct inflammatory infiltration of cardiac nerves, vagus nerves, sympathetic chain and ganglions of inferomediolateral column and hypothalamus. Other causes of bilateral facial palsy are Meningitis, Encephalitis, Malaria, Leptospirosis, Lyme disease, Sarcoidosis etc. Though bilateral facial palsy is a rare presentation in clinical medicine, but it became very important in GBS because it is an indication of impending ventilator support.

Clinical detection of bilateral facial palsy is difficult, but Bell's phenomenon, Snout reflex and emotional lability are important clinical pointers to the diagnosis of bilateral facial palsy. The diagnosis is confirmed by nerve conduction study (NCS). This study was undertaken to see the incidence of bilateral facial palsy in GBS and its importance as an indicator of severity of disease.

\section{MATERIALS AND METHODS}

21 cases of GBS admitted in different medical wards of Patna Medical College Hospital including ICU and Central Emergency were selected for this study. No. of male cases were 11 and that of female cases were 10. Diagnosis of GBS was made by detailed history, clinical examination, CSF examination and Nerve Conduction Study. The diagnosis of bilateral facial palsy was suspected by emotional lability, present bilateral Bell's phenomenon and was confirmed by NCS. Cases with other concomitant diseases like diabetes, leprosy, renal failure or any other autoimmune diseases were excluded. Cases with definite sensory level, persistent bladder and bowel dysfunction, hypokalaemia, CSF with $>50$ mononuclear cells/ mm3, abnormal porphyrin metabolism and $\mathrm{H} / \mathrm{O}$ previous polio or CVA were also excluded. The study was conducted during Jan 2017 to June 2017. Other investigations included CBC, Routine Urine Exam, Blood Sugar, Renal Function Test, Liver Function Test, Thyroid Profile, Lipid Profile, Chest x-ray PA view, ECG and ANA to exclude any other associated conditions. Cases were managed chiefly on IVIG $400 \mathrm{mg} / \mathrm{kg} /$ day for 5 days. Side effects were not seen. Cases were monitored for respiratory involvement and were transferred to ICU immediately for Ventilator support.

\section{RESULTS}

Out of 21 cases, 19 cases could be saved with loss of 1 female case with bilateral facial palsy and 1 male case without facial nerve involvement, but needing ventilator support. Hence, the mortality in this study is $9.5 \%$. No significant difference was noted in cases needing ventilator support or not, so far as the age, sex, H/O GI illness or upper limb paralysis are concerned.

\begin{tabular}{|c|c|c|c|}
\hline $\begin{array}{c}\text { No. of } \\
\text { Cases }\end{array}$ & $\begin{array}{c}\text { No. of Cases with } \\
\text { B/L Facial Palsy }\end{array}$ & $\begin{array}{c}\text { No. of Cases Needing } \\
\text { Ventilator Support }\end{array}$ & Death \\
\hline 21 & 03 & 07 & 02 \\
\hline \multicolumn{4}{|c|}{ Table 1 } \\
\hline
\end{tabular}

\section{DISCUSSION}

It is clear from the table that incidence of bilateral facial palsy in this study is $14.2 \%$. Though bilateral facial palsy is extremely rare in clinical medicine, but is common in GBS. In our study it is $14.2 \%$, but other study reports an incidence as high as $27 \%-50 \%$. Here the sample size is small, which may be the cause of showing low incidence. In other studies, cases with other causes of (in addition to GBS) bilateral facial palsy were included, but in this study established cases of GBS on the basis of clinical exam and CSF exam. (Showing raised protein and low cell count) and strict electrodiagnostic criteria such as prolongation of distal latencies $>150 \%$ of upper limit, NCV slowing $>70 \%$ of lower limit, prolongation of F-wave latencies $>150 \%$ of upper limit and reduction of CMAP amplitude $>20 \%$ of lower normal limit were included.

In our study, $33 \%$ cases needed ventilator support. It was observed that ventilator support was needed in cases with single breath count $<16$ supported by p02 $<60$ and pCO $2>40$ and chest expansion was reduced. The important observation in this study was all cases with bilateral facial palsy needed ventilator support. It is an indicator of severity of GBS and has been reported in other studies also. Another important observation was nocturnal decompensation occurring during supine position or in early morning requiring semi-elective intubation. It was most likely due to poor pulmonary mechanics, which may result up to $50 \%$ reduction in vital capacity. Secondly, impairment of central drive is another factor. $^{7}$ As reported in other studies, here in this study also no sudden death during emergency intubation was seen. Early intubation was done and there was no reason to delay intubation till blood gas value changes as the result of respiratory failure is sinister. Wijdicks et al has reported that pulmonary morbidity does not increase due to intubation where required.

\section{CONCLUSION}

As the sample size was small, the incidence of bilateral facial palsy in GBS was found to be $14.2 \%$ and most important observation was that all cases of GBS with bilateral facial palsy needed ventilator support. Hence, GBS with bilateral facial palsy should be closely monitored for respiratory functions (chest expansion, single breath count and ABG). Incidence of Guillain-Barre syndrome in both sexes were nearly equal. The percentage of cases needing ventilator support was $33 \%$ in our study. Mortality in this study was $14 \%$.

\section{ACKNOWLEDGEMENTS}

This work was supported by PMCH-Patna, a college cum hospital funded by Bihar Government, India. Dr. Ram Dayal Singh and Dr. Kumar Abhinav acknowledge PMCH-Patna for medical facilities.

\section{REFERENCES}

[1] Landry 0. Note sur la paralysie ascendante aigue. Gaz Hebd Med Chir 1859;6:472-4, 486-8.

[2] Guillain G, Barré J, Strohl A. Sur un syndrome de radiculonévrite avec hyperalbuminose du liquide céphalo-rachidien sans réaction cellulaire. Remarques sur les caractères cliniques et graphiques des réflexes tendineux. Bull Mem Soc Med Hop Paris 1916;40:1462-70.

[3] Haymaker WE, Kernohan JW. The Landry-GuilainBarre syndrome; a clinicopathological report of 50 fatal cases \& critique of the literature. Medicine (Baltimore) 1949;28(1):59-141. 


\section{Jemds.com}

[4] Fisher M. An unusual variant of acute idiopathic polyneuritis (syndrome of ophthalmolplegia, ataxia and areflexia). N Engl J Med 1956;255(2):57-65.

[5] Asbury AK, Cornblath DR. Assessment of current diagnostic criteria for Guillain-Barré syndrome. Ann Neurol 1990;27(Suppl):S21-4.

\section{Case Study}

[6] James DG. Differential diagnosis of facial nerve palsy. Sarcoidosis Vasc Diffuse Lung Dis 1997;14(2):115-20.

[7] Teitelbaum JS, Borel CO. Respiratory dysfunction in Guilain-Barre syndrome. Clin Chest Med 1994;15(4):705-14. 